\title{
A UWB Through-Wall Radar Using Beam Scanning Array Antenna
}

\author{
Fuminori Sakai $^{* 1}$, Akihiro Suzuki $^{* 1}$, Ohta Kazuo $^{* 1}$, Mituo Makimoto $^{* 1}$, Kunio Sawaya $^{* 2}$ \\ ${ }^{* 1,}$ Sakuratech Corporation, KSP E-601, 3-2-1 SAKADO, TAKATU-KU, KAWASAKI 213-0012, JAPAN \\ ${ }^{* 2}$ DEPARTMENT OF ELECTRICAL AND COMMUNICATION ENGINEERING, TOHOKU UNIVERSITY \\ AZA-AOBA 6-6-05, ARAMAKI, AOBA-KU, SENDAI 980-8579, JAPAN
}

\begin{abstract}
A UWB through-wall radar using novel beam scanning array antenna previously proposed by the authors is described. This antenna scanning system has two new technologies. First, each antenna element is equipped with an impulse generator. Second, the time control between the antenna elements is realized by using tapped delay lines and by transmitting trigger signals. Based upon above technology, experimental UWB through-wall radar having beam scanning capability is designed and constructed. The fabricated radar has compact size and light weight and is easy to use. The measurement results show excellent space resolution as a through-wall radar.
\end{abstract}

Index Terms - ultra-wideband, through-wall radar, array antenna, beam scanning, impulse.

\section{INTRODUCTION}

Ultra-wide band (UWB) radar system for automobile anticollision systems has been developed because of their capability of high space resolution, [1], [2] and has been applied to through-wall radars [3]-[5] used for antiterrorism operations. A mono-pulse receiving method for direction finding has been used mainly in both radar systems; however, this method has the following intrinsic disadvantages:

1) It is difficult to distinguish received signals scattered by two targets located at different points but having the same distance from the front of the radar. Also, there is a high probability of detecting false targets in a multipath environment.

2) Measurement of longer ranges degrades the accuracy of angle resolution. Solution of this problem requires longer spacing of the two receiving antennas, and consequently antenna size becomes larger.

3) The beam width of the antenna element must be wider than the angle resolution, which yields lower antenna gain and a shorter range of detection.

The above mentioned disadvantages can be removed by introducing the beam scanning of the transmitting antenna and/or the receiving antenna, but it brings another problem that the wideband phase shifters and controllers, which are very expensive because of their complicated circuit system and large circuit size, are required to realize the scanning UWB system. To solve these problems, a novel scanning method using a newly developed UWB impulse array antenna (IAA) has been proposed by the authors[6], [7].

In this paper, a high resolution through-wall radar with compact size and light weight is designed and constructed, and its basic performance is measured.

\section{IMPULSE ARRAY ANTENNA (IAA)}

Fig. 1 shows the basic configuration of UWB impulse array antenna (IAA) with four antenna elements. This scanning antenna is composed of antenna elements, impulse generators and delay lines. Each delay line has a fixed delay time $T_{D}$ and is driven by periodic trigger signal with an interval $T$. The beam scan angle of the array antenna is controlled by the trigger interval $T$ [6]. The waveforms of the outputs of the impulse generators are exactly the same except for the delay time of the waveforms which can be controlled easily only by varying the trigger interval $T$.

In the case of the trigger signal (a) in Fig. 1, all impulse generators are driven simultaneously because the delay time of each delay line $T_{D}$ is equal to the trigger interval $T$ and there is no phase difference between the output pulse train of the impulse generators, which yields the beam direction $\theta=0$. The trigger signal (b) in Fig. 1 is an example of the case in which the trigger interval $T$ is longer than the fixed delay time $T_{D}$. In this case, the beam direction becomes $\theta>0$. In contrast, when the delay time $T_{D}$ is longer than the trigger interval $T$, the beam direction becomes $\theta<0$. This seems to be one of the advantages of this method, i.e., beam scanning can be easily achieved by changing trigger interval $T$. The relationship among the scan angle $\theta$, the trigger interval $T$ and the delay time of each delay line $T_{D}$ is expressed by

$$
\sin \theta=\frac{T-T_{D}}{d / c}, \quad \frac{T_{D}}{2}<T<\frac{3 T_{D}}{2}
$$

Where $d$ is the spacing of array antenna and $c$ is the speed of light.

The delay time between one antenna element and the next element is expressed as the time difference between the delay time $T_{D}$ and the trigger interval $T$. This method is suitable for 
the beam steering of a wide-band antenna regardless of frequency.

Fig. 2 shows the relation between the beam direction $\theta$ and the trigger frequency calculated using equation (1). The array spacing $d$ was chosen to be $30 \mathrm{~mm}, 37 \mathrm{~mm}$, and $50 \mathrm{~mm}$ corresponding to $0.40 \lambda, 0.49 \lambda$, and $0.67 \lambda$, respectively, at 4 $\mathrm{GHz}$. It can be seen from that a beam scanning range $-40 \mathrm{deg}$ $<\theta<40$ deg can be obtained by changing the trigger frequency from $50 \mathrm{MHz}-200 \mathrm{kHz}$ to $50 \mathrm{MHz}+200 \mathrm{kHz}$, when $d=37 \mathrm{~mm}$.

\section{THROUGH-WALL RADAR DESIGN}

Based upon the fundamental discussion of IAA scanning system and the experimental verification [7], a trial throughwall radar was designed and constructed. Fig. 3 shows the block diagram of the through-wall radar using UWB IAA. The target design specifications are as follows,

System:

1) Frequency bandwidth: $3.4 \mathrm{GHz}-4.8 \mathrm{GHz}$

2) Detection range: $20 \mathrm{~m}$

3) Space resolution Distance: $25 \mathrm{~cm}$ Angle: $\quad 15 \mathrm{deg}$

4) Scanning speed: $\quad 0.5 \mathrm{sec}$
5) Display:
4.3 inch LCD

6) Size:

7) Weight:

$24.7 \mathrm{~cm} \mathrm{~L} \mathrm{x} 36.5 \mathrm{~cm} \mathrm{~W} \times 6.5 \mathrm{~cm} \mathrm{D}$

8) Supply Voltage:

$4 \mathrm{~kg}$

$5 \mathrm{~V}$

9) Signal Interface:

LAN

Hardware:

1) Transmitting antenna:

- Antenna elements: Patch antenna

- Number of antenna elements: 2 × 8

- $3 \mathrm{~dB}$ beam width

$$
\text { E-plane: } \quad 15 \mathrm{deg} \text { at } 4 \mathrm{GHz}
$$$$
H \text {-plane: } \quad 40 \mathrm{deg} \text { at } 4 \mathrm{GHz}
$$

- Array spacing: $37 \mathrm{~mm}(0.49 \lambda$ at $4 \mathrm{GHz})$

2) Receiving antenna:

- Antenna elements: Patch antenna

- Number of antenna elements: Fixed 2 x 1

- $3 \mathrm{~dB}$ beam width

$E$-plane: $\quad 72 \mathrm{deg}$ at $4 \mathrm{GHz}$
$H$-plane: $\quad 40 \mathrm{deg}$ at $4 \mathrm{GHz}$

3) Delay time: $\quad T_{D}=20 \mathrm{~ns}$ (approximately)

4) Trigger frequency: $F c=50 \mathrm{MHz}$ (center)

5) Signal detection: Correlation detector

Each Tx circuit of element in the figure is composed of a delay line, an impulse generator, a band-pass filter, and an antenna element integrated on the same substrate. The impulse generator is composed of a high-speed switching device and a driver amplifier, and the delay lines are commercially available IC's having a delay of approximately $20 \mathrm{~ns}$.

Fig. 4(a) and Fig. 4(b) show the top and the bottom view of the antenna and RF circuit pattern, respectively. As shown in the figure, the transmitting antenna is composed of $2 \times 8$ patch elements and the receiving antenna is composed of $2 \times 1$ same patch element. In the receiving circuit, a correlation detector is adopted to detect received signals. Tx and $\mathrm{Rx}$ section including antenna elements and delay lines are integrated in a multi-layered printed circuit boards.

The photograph of the trial UWB through-wall radar is shown in Fig. 5 . Small size of $24.7 \mathrm{~cm} \mathrm{~L} \mathrm{x} 36.5 \mathrm{~cm} \mathrm{~W} \times 6.5$ $\mathrm{cm} \mathrm{D}$ and light weight of $4 \mathrm{~kg}$ are realized for the portability and easy handling.

\section{EXPERIMENTS}

Prior to the measurement of radar property, experiment of Tx scanning antenna was carried out. Trigger frequency is set to be $50.0 \mathrm{MHz}$ so that the beam direction becomes $\theta=0$. The frequency of the beam direction $\theta=0$ become $46.98 \mathrm{MHz}$ because of a small deviation in the delay time of the delay lines. The measured data of the antenna pattern in azimuth angle obtained by changing trigger frequency in the range of $F c \pm 230 \mathrm{kHz}$ ( $F c$ : center frequency) in $23 \mathrm{kHz}$ increments are shown in Fig. 6, and the pattern in. It can be seen that the change in beam direction is proportional to the change in trigger frequency as indicated in Fig. 2. The measured data also show that the beam width is $\pm 18 \mathrm{deg}$ and the side lobe level is $-21 \mathrm{~dB}$ when $\theta=0$.

The experiment as the radar system was carried out through $6 \mathrm{~cm}$ thick concrete wall by detecting signals from two targets of human bodies located about $3 \mathrm{~m}$ to $4 \mathrm{~m}$ from the radar, and the transmitting antenna beam was scanned in $4 \mathrm{deg}$. step.

Fig. 8(a) and Fig. 8(b) show the measured data of the target imaging. The scattered signals from targets are displayed in the 4.3 inch LCD monitor as the radar scope image. In these figures only moving targets among various scattered signals are indicated by image signal processing. It is clearly indicated from such basic experiments that a distance resolution of $25 \mathrm{~cm}$ and an angle resolution of $15 \mathrm{deg}$ are obtained, respectively. Furthermore, Fig. 8(a) shows that it is possible to distinguish two targets located at the same distance from the radar. When the conventional mono-pulse radar is used under these measurement conditions, it is impossible to separate two signals and this is one of the notable advantage of the proposed system.

\section{CONCLUSION}

A UWB through-wall radar utilizing a newly developed electrical scanning system with impulse array antenna has been constructed and its usefulness has been experimentally verified. The transmitting array antenna is composed of 
impulse generators installed in antenna elements and tapped delay lines used to generate transmitting trigger signals. This is a considerably simplified circuit configuration. It is clearly indicated that the beam direction can be controlled from -40 deg to +38 deg by changing the trigger frequency in the range of $46.98 \mathrm{MHz} \pm 230 \mathrm{kHz}$. An evaluation of this system for a use as a through-wall radar was carried out. The obtained data proved that this radar system has excellent distance and angle resolution of $25 \mathrm{~cm}$ and $15 \mathrm{deg}$, respectively. The UWB radar system proposed here is applicable to high resolution throughwall radar with low cost, light weight and small size.

\section{REFERENCES}

[1] G. Rollmann and H. L. Bloecher, "The impact of SARA for further advances in Automotive Microwave Sensing," in Proc. of 37th European Microwave Conference, Munich Germany, Oct. 2007, pp.1523-1525.

[2] H. Veenstra, E Heijden, M. Notten, and G. Dolmans, “A SiGe-BiCMOS UWB Receiver for $24 \mathrm{GHz}$ Short-Range Automotive Radar Applications," in IEEE MTT-S Int. Microw. Symp. Dig.,Honolulu, HI, June 2007, pp.1791-1794.

[3] A. G. Yarovoy, X.Zhuge, T. G. Savelyev, and L. P. Ligthart, "Comparison of UWB Technologies for Human Being Detection with Radar," in Proc. of 37th European Microwave Conference, Munich Germany, Oct. 2007, pp.1574-1577.

[4] C. Lièbe, A. Gaugue, X. Zhao, J. Khamlichi, and M. Mènard, "A Through Wall UWB RADAR with Mechanical Sweeping System," in Proc. of 39th European Microwave Conference, Rome Italy, Oct. 2009, pp.1634-1637.

[5] A. Tan, M. Chia, and K. Rambau,"Design of Ultra-Wideband Monopulse Receiver," IEEE Trans. Microw. Theory Tech., vol.54, No.11, pp.3821-3827, Nov. 2006.

[6] F. Sakai and K. Ohta,"UWB Array Antenna Utilizing Novel Electrical Scanning System with Tapped Delay Lines," in 2010 IEEE MTT-S Int. Microw. Symp. Dig., Anaheim, CA, May 2010, pp.1262-1265.

[7] F. Sakai and K. Ohta, "An Experimental Study of Novel Scanning System Suitable for UWB Radar Application," in 2010 Asia Pacific Microwave Conference (APMC) Proceedings, Yokohama Japan, Dec. 2010, pp.1689-1692

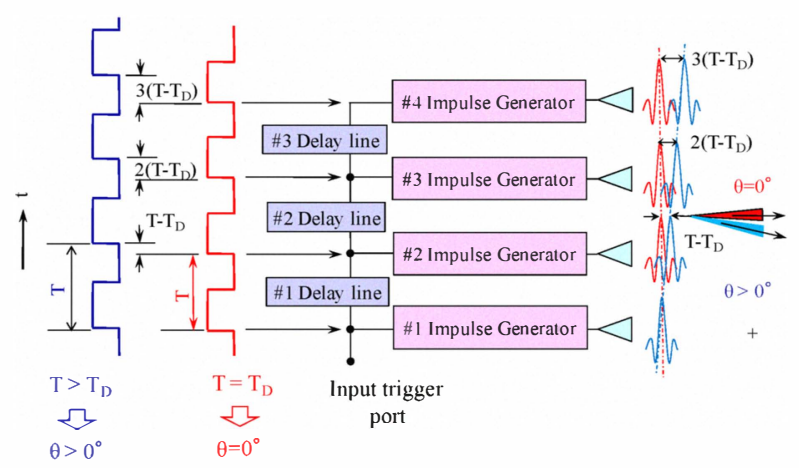

(b)

(a)

Fig. 1 Principle of impulse array beam scanning system.

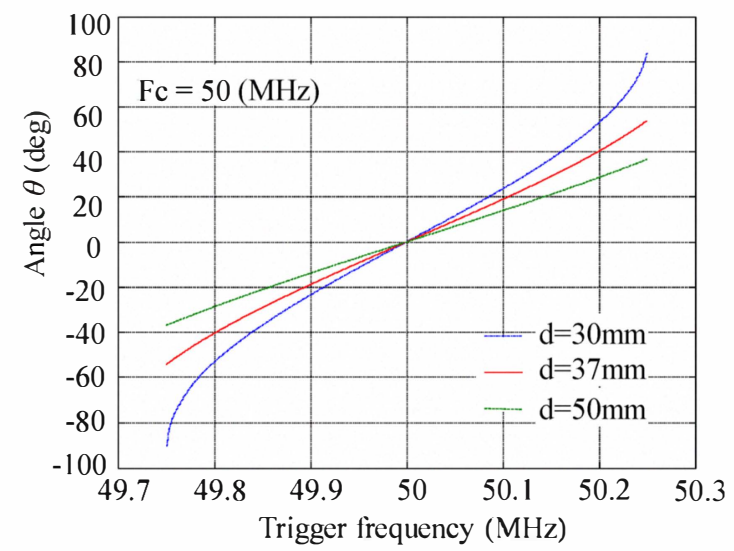

Fig.2 Relationship between beam angle and trigger frequency

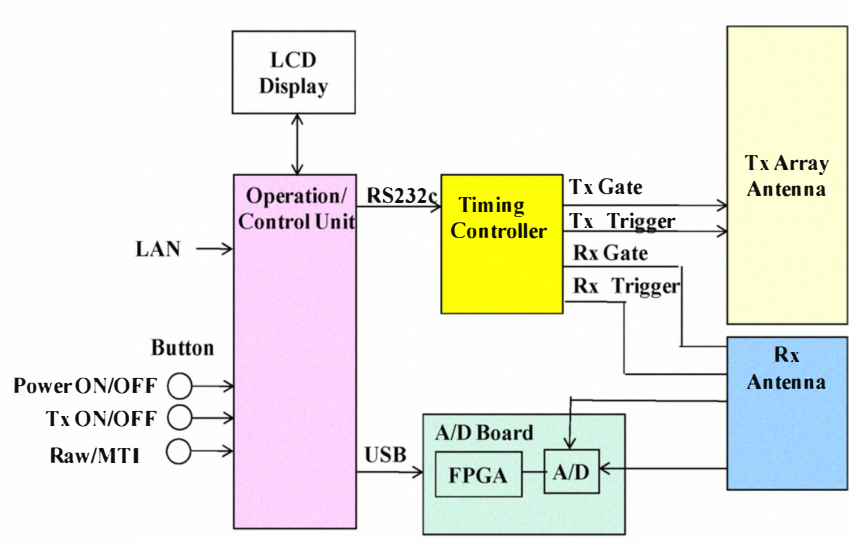

Fig. 3 Block diagram of through-wall radar.

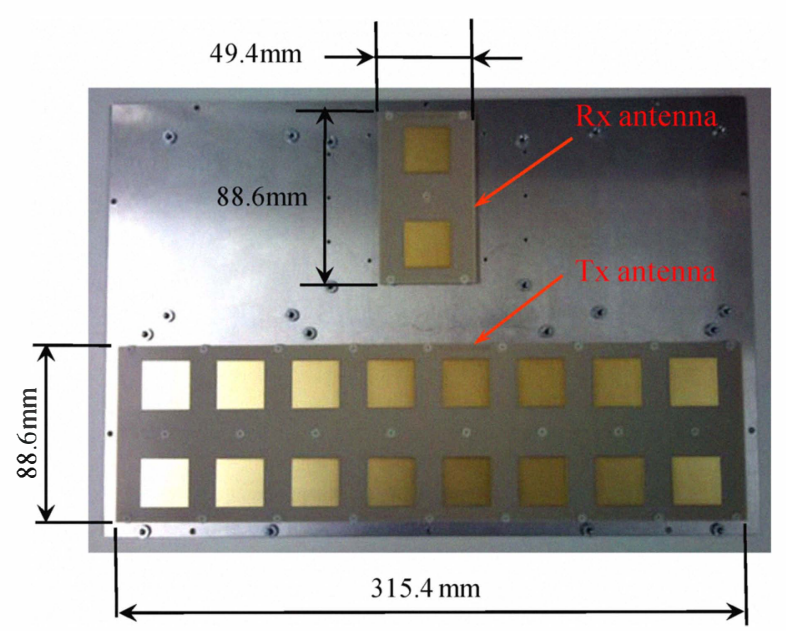

(a) Top view 


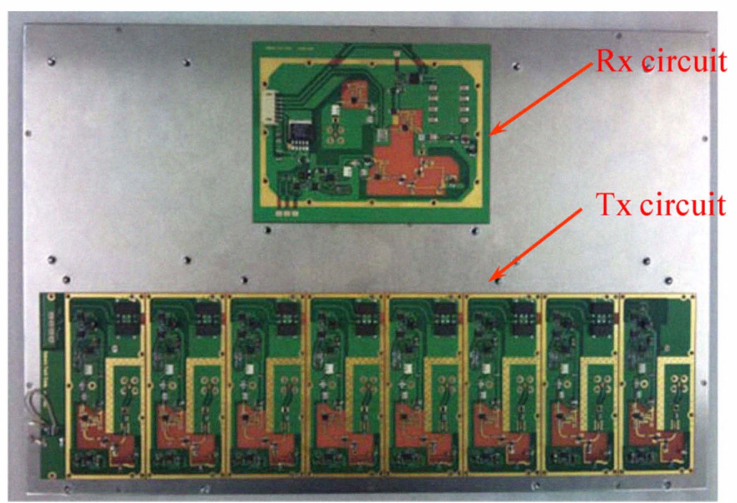

(b) Bottom view

Fig. 4 Photograph of Tx and Rx circuit.

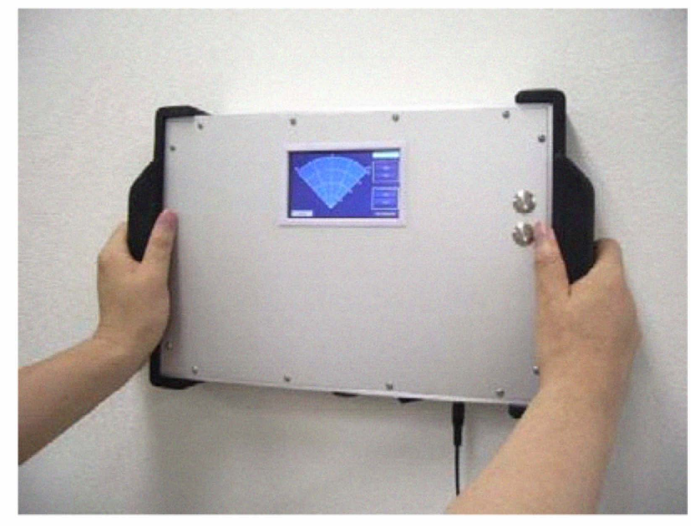

Fig. 5 The photograph of trial UWB through-wall radar.

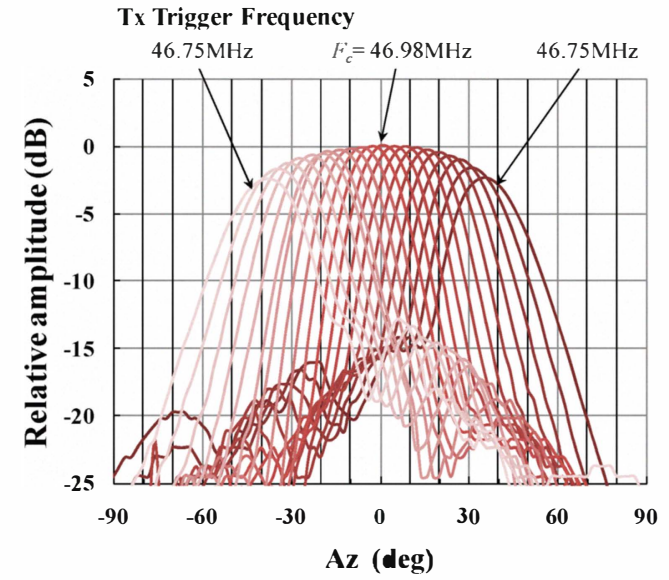

Fig. 6 Tx antenna scanning pattern in horizontal plane.

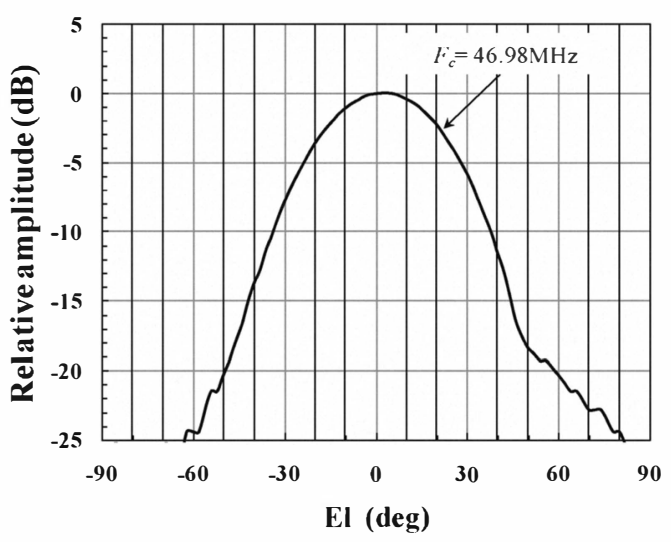

Fig. 7 Tx antenna pattern in vertical plane.

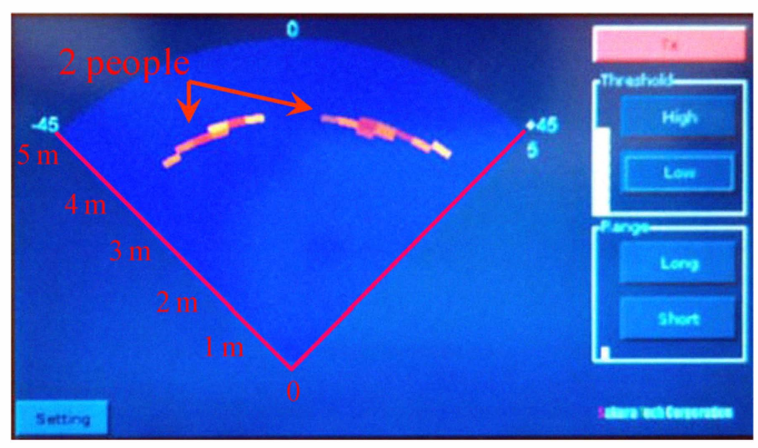

(a) Data 1

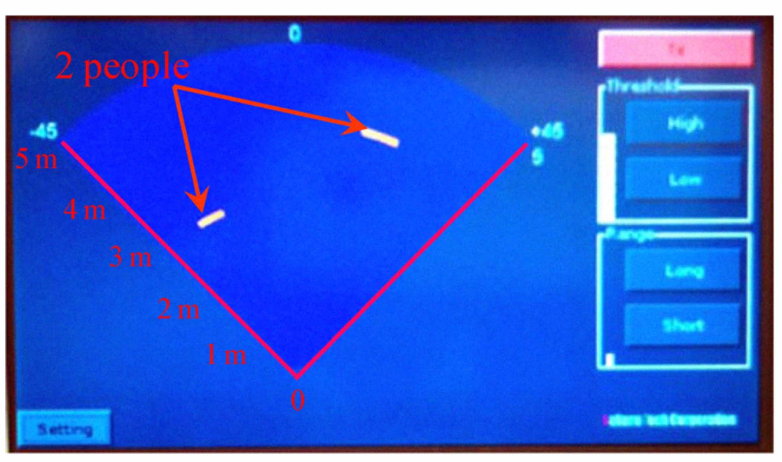

(b) Data 2

Fig. 8 Target image. 